\title{
HYDROGEN INTERACTION WITH EUVL-RELEVANT OPTICAL
}

\section{MATERIALS}

\author{
A.S. Kuznetsov ${ }^{1}$, R.W.E van de Kruijs ${ }^{1}$, M.A. Gleeson ${ }^{1}$, K. Schmid ${ }^{2}$, F.Bijkerk ${ }^{1}$ * \\ ${ }^{1}$ FOM Institute for Plasma Physics Rijnhuizen, Nieuwegein, The Netherlands \\ ${ }^{2}$ Max-Planck Institut für Plasmaphysik, Boltzmannstraße 2, 85748 Garching, Germany \\ * also at MESA+ Institute for Nanotechnology, Enschede, The Netherlands
}

\begin{abstract}
$\mathrm{Mo} / \mathrm{Si}$ multilayer mirrors were exposed to deuterium ions with well-defined energies in order to gain insight into the effects of Extreme UV light driven plasma generation on reflective elements in commercial lithography equipment. Post-irradiation analysis by Rutherford Backscattering Spectrometry (RBS) showed erosion of both Mo and Si layers for the highest energy (50 eV/D) and exposure time $\left(5.4 \times 10^{4} \mathrm{~s}\right)$. Nuclear Reaction Analysis (NRA) revealed detectable deuterium retention for energies $\geq 25 \mathrm{eV} / \mathrm{D}$. Surface analysis by X-ray Photoelectron spectroscopy (XPS) showed erosion of the first Si layer for energies $\geq 5 \mathrm{eV} / \mathrm{D}$. Inferences on the spatial distribution of trapped deuterium are made on the basis of available data regarding deuterium retention in the materials in question.
\end{abstract}

\section{Introduction}

Production of multilayer mirrors for Extreme UV lithography (EUVL) requires understanding and control of the physical processes inside and at the surface of the EUV optics. Such optics consists of multilayer coatings designed to reflect the wavelength of 
interest. They are required to be fabricated with an accuracy on the picometer level. Progress in the development of deposition equipment and procedures has ensured that multilayers fulfilling all optical requirements can be routinely deposited $[1,2]$. However, for application in a commercial EUVL environment, there still remains the challenge of fast degradation of optics under the intense power loads and/or repetitive cleaning cycles based on the use of hydrogen. Under these conditions there are a variety of processes that can jeopardize the lifetime of the optics, including photo-chemistry induced contamination, thermally induced inter-diffusion of the multilayer structure, and the interaction of radiation-driven plasma with mirrors.

The chemical composition of the ambient environment is an important factor in determining the lifetime of the optical components. Hydrogen is currently under consideration as a buffer gas in lithography machines in order to suppress tin contamination from the EUVL source. It may also have beneficial effects by, for instance, inducing a self-cleaning process at the optical surfaces and reversing oxidation processes at the surfaces [3]. However, its usage also has potential drawbacks. Among the negative consequences that may arise, hydrogen treatment of the optics may result in retention in the multilayer structure, resulting in swelling and changes to the inter-layer spacing, thus altering the optical properties of the structure. Other processes that may occur include erosion due to energetic (plasma-sheath accelerated) hydrogen ions, enhanced inter-layer mixing and inter-diffusion, acceleration of chemical processes at interfaces due to presence of hydrogen. Some of these processes have been observed in various two-component structures. For example, enhanced diffusion was observed in PdPt or Ni-Cu alloys during annealing in hydrogen atmosphere [4,5]. 
The aim of our on-going research is to study the interaction of hydrogen (atoms and ions) with materials that are optically relevant for EUVL, including associated processes such as interactions involving oxygen and nitrogen. The overall goal is to establish a model and obtain the required understanding of (photo-)induced processes involving hydrogen, oxygen and nitrogen at EUVL-relevant surfaces, such as multilayer materials (e.g. Mo and $\mathrm{Si}$ ) as well as capping layer materials. In this paper we focus on the interaction of energetic deuterium ions with standard, uncapped multilayer mirror surfaces.

\section{Experimental}

Experiments were conducted to study the influence of hydrogen on real multilayer systems. Samples were prepared, based on magnetron sputtering technique, at the inhouse deposition facilities at FOM Rijnhuizen. The samples studied consisted of 50 bilayers of molybdenum and silicon with thicknesses of 3 and $4 \mathrm{~nm}$ respectively. The outermost layer was Si.

Irradiation of these samples and some post-exposure analytical procedures were conducted at the Max-Planck Institut für Plasmaphysik in Garching, Germany. The sample irradiations were performed in the 'PLAQ' set-up, which uses an electroncyclotron resonance plasma. A detailed description of the set-up and associated analysis facilities can be found elsewhere [6,7]. The samples were irradiated with low-energy deuterium $\left(\mathrm{D}_{3}{ }^{+}\right)$ions $(5,25$ and $50 \mathrm{eV} / \mathrm{D})$, with a flux of typically $1 \times 10^{15} \mathrm{D} / \mathrm{cm}^{2} \mathrm{~s}$. Total exposure fluences of up to $\sim 3 \times 10^{20} \mathrm{D} / \mathrm{cm}^{2}$ were used. Post-irradiation Rutherford Backscattering Spectrometry (RBS) and Nuclear Reaction Analysis (NRA) 
measurements were performed to track changes in the samples. Subsequent X-ray Photoelectron Spectroscopy (XPS) analysis was done at FOM Rijnhuizen.

\section{Results and discussion}

With the exception of the sample exposed to the highest energy for the longest time (50 eV/D ions for $5.4 \times 10^{4} \mathrm{~s}$ ), no significant changes in the RBS spectra were observed for the irradiated samples. A comparison of the RBS spectra from the un-irradiated sample and the sample exposed to $50 \mathrm{eV} / \mathrm{D}$ ions for $5.4 \times 10^{4} \mathrm{~s}$ is shown in Fig.1. The changes observed in the RBS spectra in this case are most likely related to the presence of low levels of impurities in the beam, due to the fact that both Mo and Si layers were eroded even though the energy used is below the sputtering threshold of molybdenum by deuterium. The features in the spectra (going from the right side) represent Mo layers, Si layers and the wafer respectively. Erosion is evident from the shift of the spectrum to the right.

Results of the post-irradiation NRA analysis are shown in Fig.2. For the $5 \mathrm{eV} / \mathrm{D}$ exposures (not shown) there was a slight increase in the NRA signal as compared with the un-irradiated samples. However, this was at the limit of detection $\left(10^{12} \mathrm{D} / \mathrm{cm}^{2}\right)$. Thus, no firm conclusion can be made regarding D retention at this energy. In cases of $25 \mathrm{eV} / \mathrm{D}$ and $50 \mathrm{eV} / \mathrm{D}$ irradiations deuterium retention is on the order of $1 \times 10^{16} \mathrm{D} / \mathrm{cm}^{2}$ for all exposed samples. There is no obvious substantial change in the overall level of D retention on either the energy or the exposure time. The surface region appears to have already saturated after $6000 \mathrm{~s}$ exposure and there is no significant difference between irradiation at 25 and $50 \mathrm{eV} / \mathrm{D}$. 
To confirm the state of the surface after irradiation, analysis of surface composition was conducted via XPS. Comparison of a survey XPS spectra from an un-irradiated sample and from a sample exposed to $5 \mathrm{eV} / \mathrm{D}$ ions are shown in Fig. 3. One can see major changes in intensities of Mo $3 \mathrm{~d}$ and $\mathrm{Si} 2 \mathrm{p}$ peaks after irradiation. It was found that even after irradiation with $5 \mathrm{eV} / \mathrm{D}$ ions the first silicon layer was severely damaged as illustrated by the increased molybdenum content at the surface. Quantification of the main surface elements based on XPS measurements are shown in Fig.4. The left-most (0 eV) data-points are obtained from an un-irradiated sample. The silicon concentration on the surface decreased from $50 \%$ to $\sim 3 \%$ while the molybdenum concentration increased from $3 \%$ to $\sim 15 \%$. This illustrates the silicon depletion from the surface irrespective of the particle energy. Since the sputtering threshold for deuterium bombardment of silicon is approximately $30 \mathrm{eV}[8]$, the results suggest that chemical erosion is an important mechanism for silicon removal in the case of $\mathrm{H}$ irradiation.

The question arises as to where the trapped deuterium is located. We simulated the bombardment process using TRIM, accounting for the erosion of the first silicon layer, in order to estimate the approximate penetration depth of the $\mathrm{D}$ ions. The calculations indicated that $25 \mathrm{eV} / \mathrm{D}$ and $50 \mathrm{eV} / \mathrm{D}$ atoms can only penetrate into the first molybdenum layer. Based on the NRA profiles of deuterium retention from [7], where polycrystalline Mo films were irradiated with deuterium ions, one can make an estimate of the amount of D that can be retained by this layer. From [7], the highest atomic concentration of D measured in polycrystalline Mo was $\sim 6 \%$. Assuming that this value represents the upper limit for D retention in Mo, it gives a value of $\sim 10^{15} \mathrm{D} / \mathrm{cm}^{2}$ for the $3 \mathrm{~nm}$ layer. Note that in [7] $200 \mathrm{eV} / \mathrm{D}$ ions were used, so this data might not be representative of the $\mathrm{D}$ retention as a result of 25 and $50 \mathrm{eV}$ exposures. 
The samples irradiated with energies 25 and $50 \mathrm{eV} / \mathrm{D}$ contain $\sim 10^{16} \mathrm{D} / \mathrm{cm}^{2}$ based on the NRA data. Hence, retention by an outermost Mo layer is insufficient to explain the results obtained (on the assumption that $6 \%$ does indeed represent the saturation value). In this case it is necessary to infer a diffusion processes and that the majority of deuterium is trapped in the second silicon layer or deeper. If we assume all the D indicated in figure 2 is trapped in a single $\mathrm{Si}$ layer, then the resultant deuterium to silicon atomic ratio in that layer is approximately 0.4 . This value is consistent with the highest hydrogen content obtain by Schlatmann et al. [9]. Hence, such a D content is at least physically possibly. Considering the data from [9], where $\mathrm{Mo} / \mathrm{Si}$ multilayers were grown with each Si layer being bombarded by hydrogen ions, this deuterium content represents a $\sim 70 \%$ density decrease relative to the density of crystalline Si. This leads to the possibility that, as was experimentally reported in [9], the Si layer was swollen. If this is the case, low-energy hydrogen ion irradiation of multilayers will result in a reflectivity drop due to the changed inter-layer spacing. This possibility requires further investigation.

\section{Conclusions}

In this work we presented first results on hydrogen interaction with EUVL relevant materials. Irradiations of $\mathrm{Mo} / \mathrm{Si}$ multilayer structures with low-energy deuterium ions were conducted. Analysis has shown erosion of the first silicon layer for ion-energies $\geq 5$ $\mathrm{eV} / \mathrm{D}$ and a deuterium retention of approximately $1 \times 10^{16} \mathrm{D} / \mathrm{cm}^{2}$ for energies $\geq 25 \mathrm{eV} / \mathrm{D}$. The penetration depth of ions and the low retention of deuterium by molybdenum suggests that the majority of deuterium diffuses to the layers underneath the 
molybdenum. If all the $\mathrm{D}$ is retained in the Si-layer immediately beneath the outermost Mo layer, then it represents a deuterium atomic content of $\sim 40 \%$.

Further investigation, including modeling, will be directed to subsurface processes caused by deuterium trapping in the sample. New experiments are planned to investigate the influence of hydrogen impact on multilayers using higher fluences, closer to 'full lifetime fluence' of the lithography optics (approximately $10^{21}-10^{22} \mathrm{ion} / \mathrm{cm}^{2}$ ). Irradiation of multilayers with hydrogen atoms is scheduled to investigate the influence of chemical sputtering effects.

\section{Acknowledgements}

This work is part of FOM Industrial Partnership Programme I10 ('eXtreme UV Multilayer optics', or 'XMO') with additional funding from SenterNovem through the 'ACHieVE' programme.

We would like to thank Dr. Erwin Zoethout for conducting XPS measurements, Frenk Boekhout and the ADC team for sample preparations, Saskia Bruijn and Toine van den Boogaard for help in data analysis. 


\section{References}

1. Louis E., Yakshin A.E., Zoethout E., van de Kruijs R.W.E. , Nedelcu I., Alonso van der Westen S., Tsarfati T., Bijkerk F., Enkisch H., Müllender S.,Wolschrijn B., Mertens B. // SPIE Proceedings, 2005. V. 5900.

2. Yakshin A.E., van de Kruijs R.W.E., Nedelcu I., Zoethout E., Louis E., Bijkerk F. // SPIE Proceedings, 2007. V. 6517.

3. Tsarfati T., Zoethout E., van de Kruijs R.W.E., Bijkerk F. // Surface Science, 2009. V. 603. P. 2594.

4. van den Oetelaar L.C.A., Nooij O.W., Oerlemans S., Denier van der Gon A.W., Brongersma H.H., Lefferts L., Roosenbrand A.G., van Veer J.A.R. // J. Phys. Chem. B, 1998. V.102. P. 3445.

5. Fukai Y., Mizutani M.,Yokota S., Kanazawa M., Miura Y.,Watanabe T. // J. Alloys Comp., 2003. V.356-357. P. 270.

6. Landkammer B., von Keudell A., Jacob W. // J.Nucl. Mater.,1999. V. 264. P. 48.

7. Alimov V. Kh., Roth J., Lindig S. // J. Nucl. Mater., 2008. V. 381. P. 267.

8. Yamamura Y., Tawara H. // Atomic Data and Nuclear Data Tables,1996. V. 62. P.149.

9. Schlatmann R., Keppel A., Xue Y., Verhoeven J. // J. Appl. Phys., 1996. V. 80. P. 2121 
Fig.1. RBS analysis of an un-irradiated sample (solid line) and a sample with the highest exposure time at $50 \mathrm{eV} / \mathrm{D}$ (dotted line)

Fig.2. Deuterium retention in the multilayer structure for different exposure doses and ion-energies, measured via NRA

Fig.3. Survey XPS spectra of a un-irradiated sample and a sample irradiated with $5 \mathrm{eV} / \mathrm{D}$ Fig.4. Surface composition as a function of incident ion-energy, based on XPS measurements 
Fig. 1.

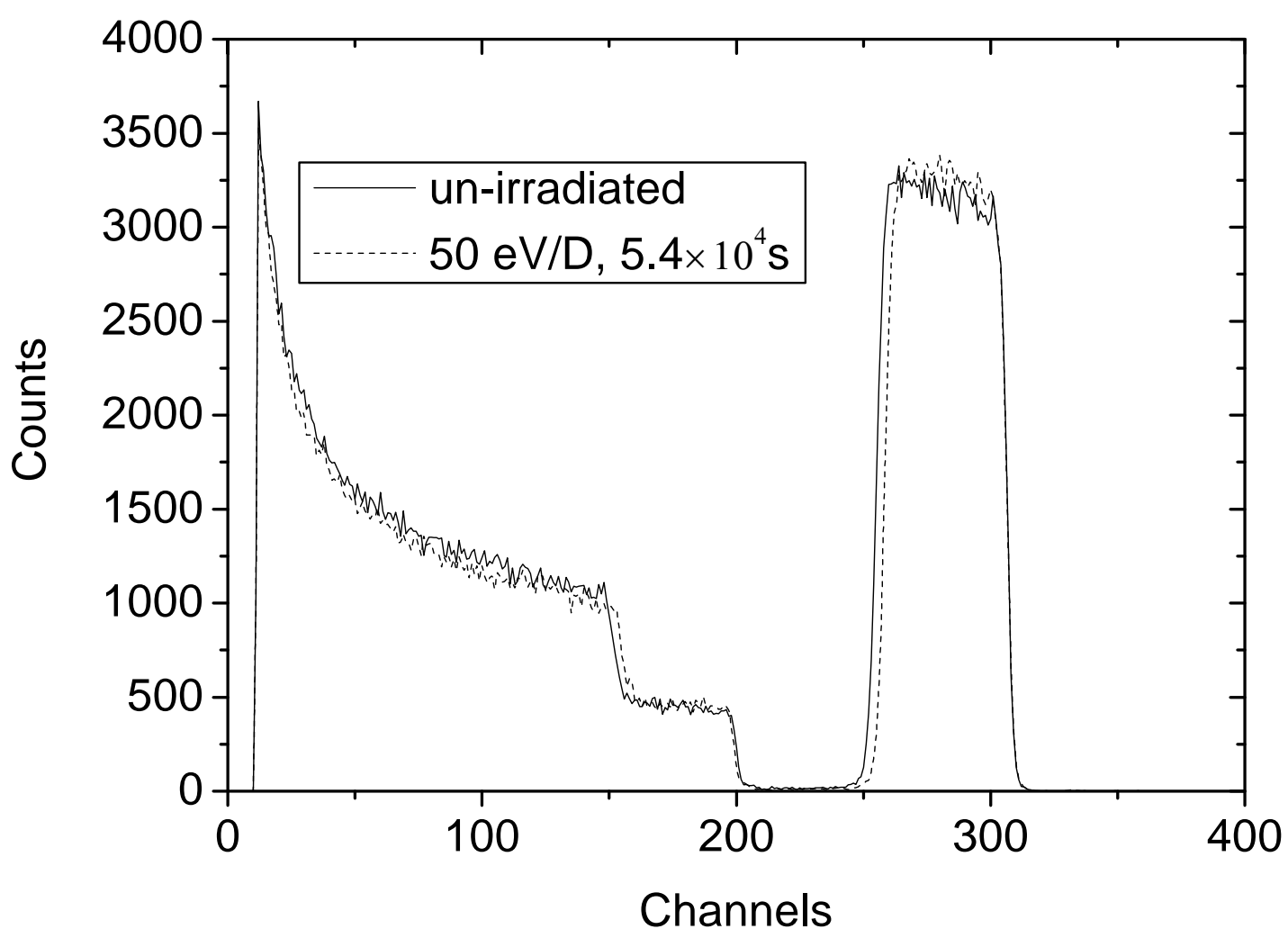


Fig. 2.

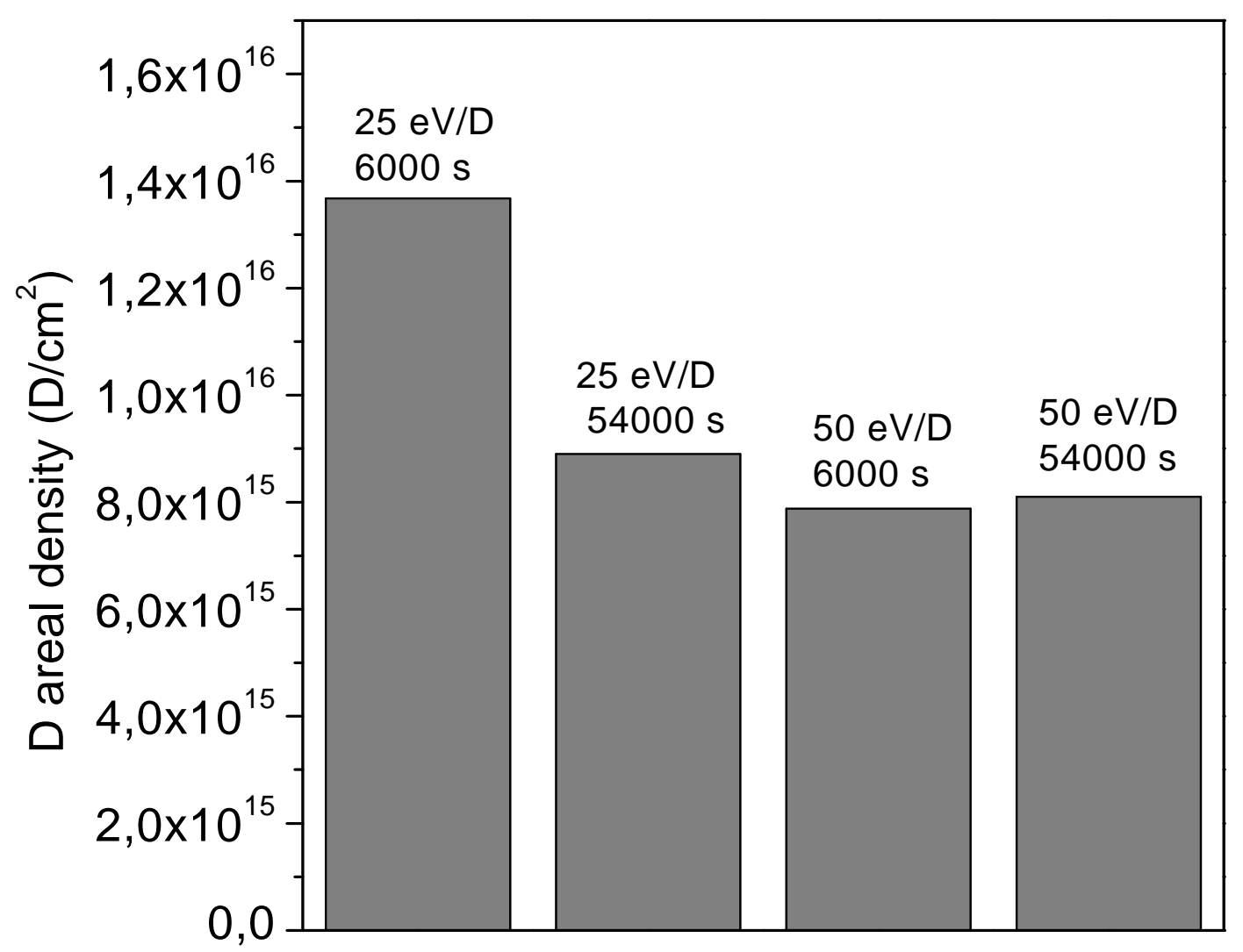


Fig. 3.

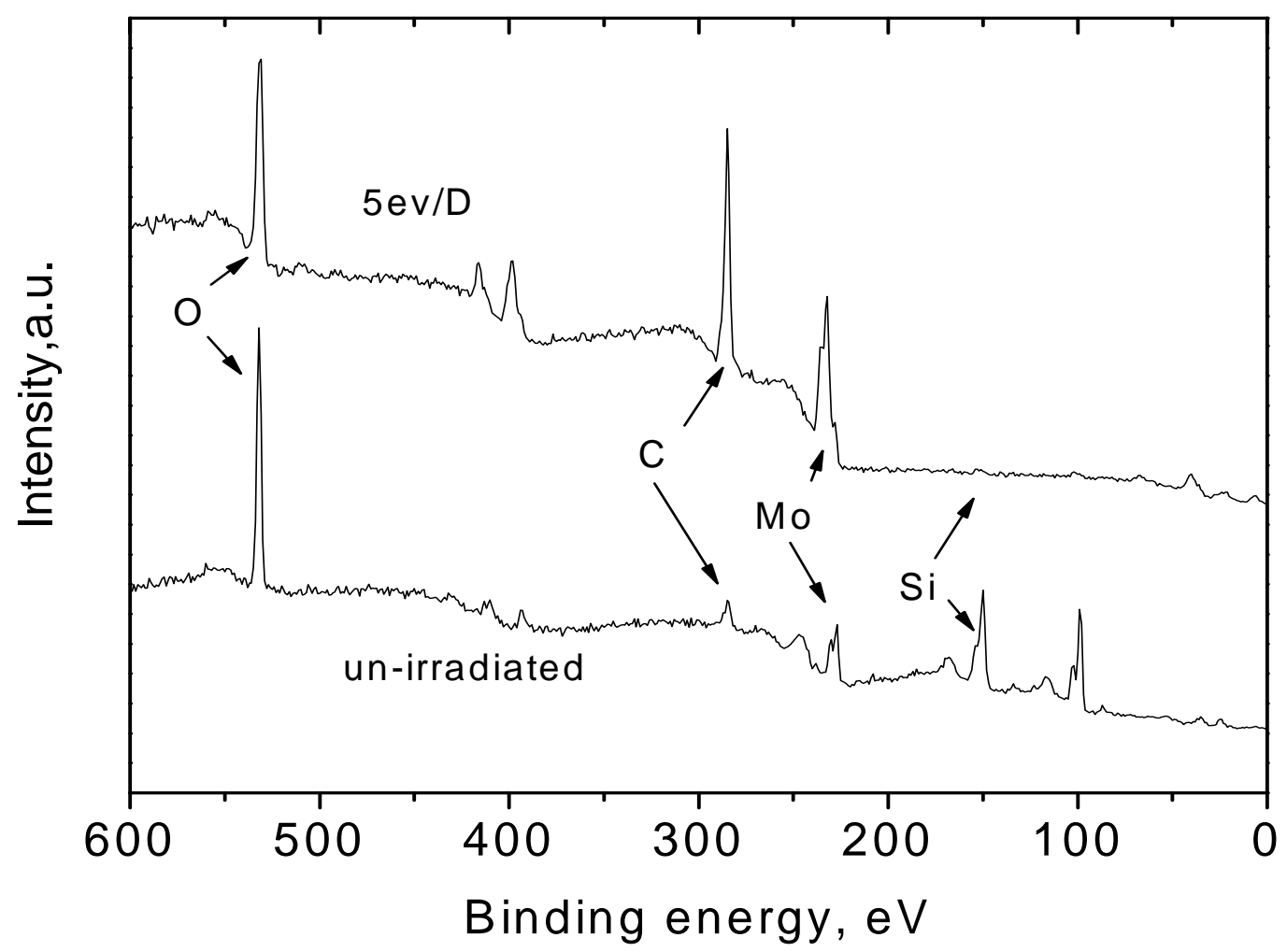


Fig. 4.

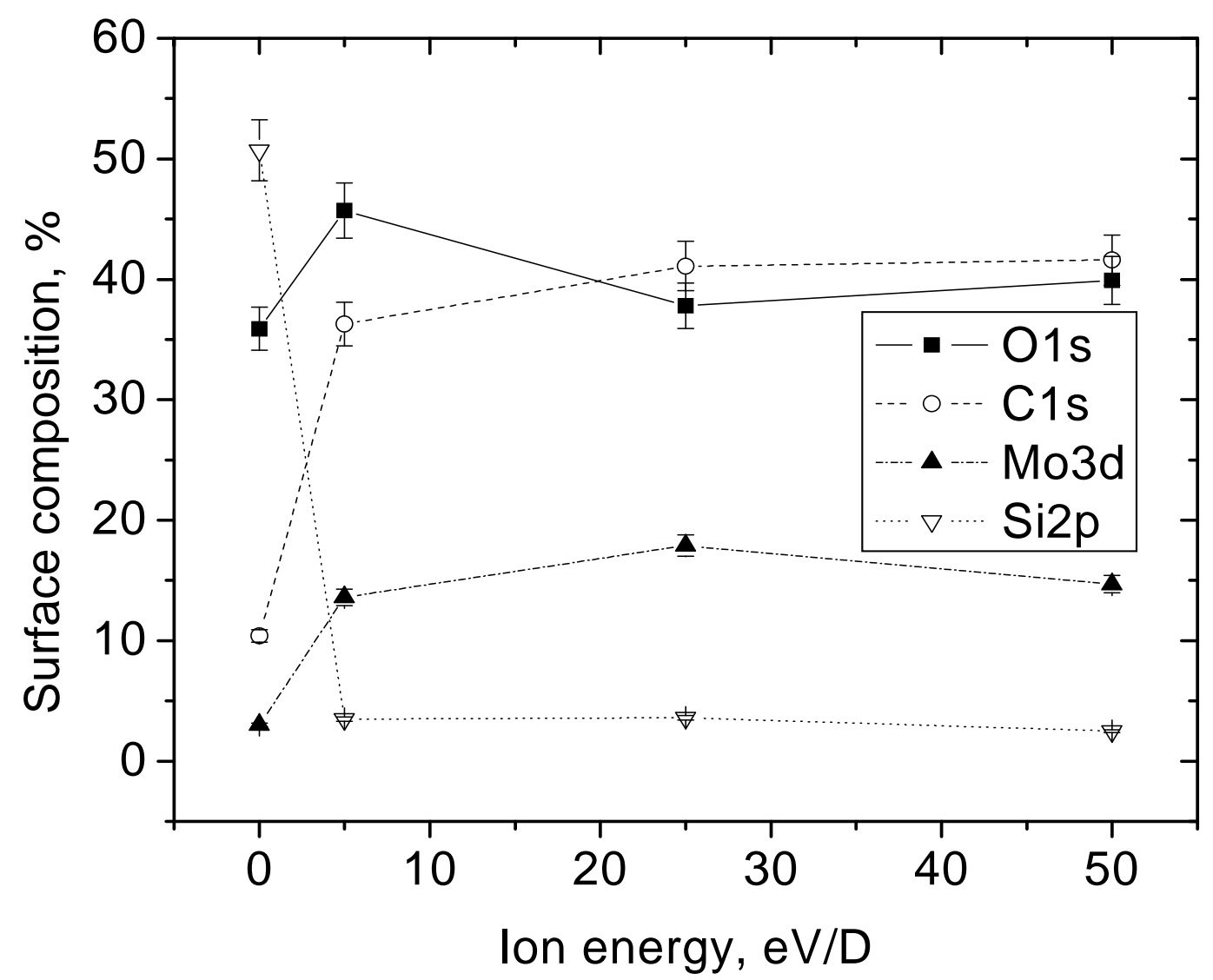


Authors

1. Alexey Kuznetsov, Edisonbaan 14, 3439 MN Nieuwegein, the Netherlands.

E-mail: $\underline{\text { A.Kuznetsov@rijnhuizen.nl }}$

2. Robbert W.E. van de Kruijs, Edisonbaan 14, 3439 MN Nieuwegein, the Netherlands. E-mail: R.W.E.vandeKruijs@Rijnhuizen.nl

3. Michael Anthony Gleeson, Edisonbaan 14, 3439 MN Nieuwegein, the Netherlands. E-mail: M.A.Gleeson@,rijnhuizen.nl

4. Klaus Schmid, Boltzmannstraße 2, 85748 Garching, Germany. E-mail: Klaus.Schmid@,ipp.mpg.de

5. Fred Bijkerk, Edisonbaan 14, 3439 MN Nieuwegein, the Netherlands. E-mail: F.Bijkerk@rijnhuizen.nl 